\title{
Endovascular treatment of complex intracranial aneurysms
}

\author{
Mariusz Hofman ${ }^{1 A, B, C, D, E, F}$, Tomasz Jamróz ${ }^{1 A, B, C, D, E, F}$, Izabela Jakutowicz ${ }^{1 A, B, C, E}$, Paweł Jarski ${ }^{1 B, D, E}$, \\ Wilhelm Masarczyk ${ }^{1 B, D, E}$, Marcin Niedbała ${ }^{1 B, D, E}$, Nikodem Przybyłko ${ }^{1 C, E, F}$, Damian Kocur ${ }^{1 E, F}$, Jan Baron ${ }^{2 A, F}$
}

'Department of Neurosurgery, Medical University of Silesia, Katowice, Poland

2Department of Radiology and Nuclear Medicine, Medical University of Silesia, Katowice, Poland

\section{Abstract}

Purpose: Complex intracranial aneurysms (CIA) are heterogenous group of intracranial vascular malformations. Due to its giant size, difficult location, broad neck, branches arising from the aneurysm, wall structure, calcification, presence of intraluminal thrombus or previous treatments it requires more careful approach. The aim of this study was to evaluate endovascular treatment results of CIA in our Department.

Material and methods: In order to differentiate CIA from all the aneurysms, treated endovascularly in years 2008-2014, authors proposed their own qualification criteria. Additionally, subgroup of patients with CIA with simultaneous subarachnoid haemorrhage (SAH) was divided. Clinical outcomes of patients were assessed with Glasgow Outcome Scale (GOS), while radiological outcomes were assessed with Montreal Scale. Aneurysm localization, incidence of aborted procedures, intraoperative complications were also evaluated.

Results: Internal carotid artery was the most common localization in both CIA and non-complex (nCIA) groups. Incidence of aborted procedures was significantly higher in CIA group than in nCIA (25\% vs. $7 \%$; $p<0.01)$. CIA group had worse Montreal scores then nCIA group (1.90 vs. 1.49; $p<0.01$ ). Clinical outcome in GOS scale in patients with SAH and CIA was significantly worse than in SAH and nCIA (2.86 vs. $4.06 ; p=0.04$ ).

Conclusions: To conclude, proposed criteria of CIA should be taken into consideration during diagnosis and qualification to invasive treatment. Classifying aneurysm as CIA is related to greater possibility of aborting endovascular procedure due to technical difficulties.

Key words: aneurysm, complex, morphology, embolization.

\section{Introduction}

Treatment of intracranial aneurysms is an ever-improving field of modern neurosurgery and interventional neuroradiology. Among the most commonly used treatment techniques are clipping and endovascular embolisation, which can be assisted by the use of balloons or stents [1,2]. Bypass surgery coupled with clipping of an aneurysm's parent vessel can be used with success as a last resort as well [3-5]. Modern techniques of intracranial aneurysm treat- ment reduce the hospitalisation period, are usually linked with lower risk of complications, and allow the treatment of more demanding aneurysms.

The therapeutic team composed of neurosurgeons and neuroradiologists should always individualise the treatment process, taking into account the specific aneurysm characteristics, patient's state, and a spectrum of other factors such as the experience of the particular departments [6]. Nowadays, those two methods are increasingly recognised as complementary rather than competitive [7]. This

Correspondence address:

Mariusz Hofman, Katedra i Klinika Neurochirurgii Śląskiego Uniwersytetu Medycznego w Katowicach, 14 Medyków St., 40-752 Katowice, phone +4832 7894501 , e-mail:mariuszhf@gmail.com

Authors' contribution:

A: Study design, B: Data dollection, C: Statistical dnalysis, D: Data interpretation, E: Manuscript preparation, F: Literature search, G: Funds collection 
kind of approach should be preceded by precise diagnostic procedures, especially imaging techniques including computed tomography angiography, digital subtraction angiography (DSA), or 3D rotational angiography. This increases the chance of therapeutic success.

In the past few years, the authors of multiple publications tried to separate a group of complex intracranial aneurysms (CIA), which could be linked with worse treatment outcomes [8-12]. Andaluz and Zuccarello, in an article published in 2011, tried to specify some of CIA's features, such as: giant size, difficult location, broad neck, branches arising from the aneurysm wall structure, calcification, the presence of intraluminal thrombus, or previous treatments [11]. So far there has been a lack of consensus regarding which aneurysms should be classified as CIA [9-11]. It is a serious issue that makes it difficult to compare research articles on this subject, and consequently physicians do not possess a tool for clinical assessment, initial prognosis, and patient outcome after treatment of CIA. The authors present the results of endovascular treatment in our department for aneurysms with chosen morphological features that could classify them as CIA.

\section{Material and methods}

\section{Patient population}

In this analysis 354 patients with average age 54.05 years $(\sigma=10.75)$ were diagnosed with intracranial aneurysms and were qualified for endovascular treatment in our centre (Table 1) during the years 2008-2014. In total, 303 patients in our database were treated for the first time, and 51 patients had been treated with neurosurgical clipping or endovascular embolisation previously. The examined population comprised of 269 (75.99\%) women and $85(24.01 \%)$ men. The possible course of treatment was chosen based on patients' clinical presentation and DSA

Table 1. Summary of patients' characteristics

\begin{tabular}{|l|c|}
\hline Factor & $n=354$ \\
\hline First treatment & 303 \\
\hline Second or subsequent treatment & 51 \\
\hline Men & 85 \\
\hline Women & 269 \\
\hline Age (year), mean & 54.05 \\
\hline
\end{tabular}

Table 2. Evaluation of aneurysmal occlusion directly after embolisation and in follow-ups

\begin{tabular}{|l|c|}
\hline Montreal Scale & \\
\hline 1 & Complete embolisation \\
\hline 2 & Residual neck \\
\hline 3 & Residual dome \\
\hline
\end{tabular}

imaging. This decision was made by an interdisciplinary team composed of neurosurgeons and interventional neuroradiologists. Patients with incomplete medical records were eliminated from the database and did not participate in this study.

\section{Clinical data}

Medical records were reviewed for all the patients. In the clinical database, the patients' status on admission to the hospital was evaluated. Comorbidities, neurological symptoms, Glasgow Coma Scale (GCS) score, and the presence or absence of subarachnoid haemorrhage (SAH) were assessed. The patients' condition with ruptured aneurysm was rated using Fisher and Hunt-Hess scales. The clinical data contained also information about the type and time of the first imaging study in which the aneurysm was diagnosed. The interruption of the procedure and the reasons for aborting the procedure were assessed. The necessity of stent implantation was also evaluated. The database also included information on the duration of stay in the neurosurgery ward and intensive care unit (ICU) after the intervention, intraoperative complications, and perioperative mortality. The clinical status of patients was evaluated using the Glasgow Outcome Scale (GOS).

\section{Imaging data}

Each patient qualified to this study had undergone 3-dimentional digital subtraction angiography (3-D DSA). In the study, the following aneurysm parameters were evaluated: length and width of the neck; length, width, and height of the dome of the aneurysm; and localisation of intracranial aneurysms. Based on the above-mentioned parameters, bottleneck factors were determined. The bottleneck factor is defined as the dome-to-neck ratio. In the literature it is not specified which size of dome and which size of neck is taken into account in the estimation; therefore, in our study the following different modifications were checked: length of dome to length of neck (BNL), width of dome to width of neck (BNW), maximum dimension - maximum dome size to minimum neck size $\left(\mathrm{BN}_{\max }\right)$, minimum dimension - minimum dome size to maximum neck size $\left(\mathrm{BN}_{\text {min }}\right)$, average bottleneck (average value of $\mathrm{NBL}, \mathrm{NBW}, \mathrm{BN}_{\max }$ and $\mathrm{BN}_{\text {min }}$ ), and average size of the aneurysms - it was calculated as an arithmetic average of length, width, and height of the dome.

In order to evaluate the effectiveness of the procedures the Montreal Scale (Table 2) was used. In our centre, aneurysmal occlusion was assessed based on 3-D DSA directly after the intervention and also in follow-ups based on 3-D DSA and angio-CT. Cases of impossible assessment of aneurysmal occlusion in angio-CT due to the presence of artefacts could not be taken into consideration. Follow-ups were carried out at least six months 
after the procedure. In our study, the results of treatment collected up to the end of 2016 were analysed.

\section{Complex intracranial aneurysms - qualification}

In our study, patients with CIA were selected from the examined population. The parameters of complexity were established to qualify the aneurysm to the above-mentioned group. Size of aneurysm, bottleneck factor, and width of aneurysm neck were evaluated. If either the length, width, or height were more than $25 \mathrm{~mm}$ and the average bottleneck factor was below 1 or the width of the aneurysm neck was above $10 \mathrm{~mm}$, the aneurysm was included into the group of aneurysms of complex morphology. Other inclusion criteria were: previous history of neurosurgical clipping, presence of arteries arising from the dome, and difficult morphology (e.g. unfavourable anatomical conditions concerning parental artery or direction of the aneurysm).

\section{Results}

\section{Characteristics of aneurysms}

Endovascular embolisation was performed in 354 patients with intracranial aneurysm. Forty (11.30\%) of them were classified as aneurysms of complex morphology. CIA were selected basing on six qualifying factors (Table 3).

The most common localisation for CIA was the internal carotid artery (ICA) -22 aneurysms $(55.00 \%)$. Based on the analysis of the whole examined population, ICA was also the most common localisation of non-complex intracranial aneurysms (nCIA) (Table 4).

\section{Analysis of clinical data}

The analysis of our database has shown that the presence of SAH was similar for both groups: seven (17.50\%) for patients with CIA and 77 (24.52\%) for patients with aneurysms of non-complex morphology $(p>0.05)$. Intraoperative complications: haematoma at the injection site, ischaemic stroke, rupture of aneurysm during procedure, and intracranial haematoma were present in both groups with comparable frequency (Table 5).

In the terms of the mortality of CIA and nCIA patients who underwent the endovascular treatment, there were no statistically significant differences $(p>0.05)$. Among patients with CIA, we recorded two cases of death (both as natural course of the disease), which constituted $5 \%$ of this group. In the group of patients with nCIA the mortality was at the level of $4.77 \%$; we recorded 15 deaths. Seven patients died as a natural course of the disease, while eight died due to intraprocedural complications (three suffered from ischaemic stroke, in five cases intraoperative rupture of aneurysm occurred).

The study showed that the group of patients who were discharged with a maximum GOS score of 5 was smaller
Table 3. Number of aneurysms qualified as complex intracranial aneurysms according to qualifying factors

\begin{tabular}{|l|c|}
\hline Qualifying factor & $\begin{array}{c}\text { Population of complex } \\
\text { aneurysms }\end{array}$ \\
\hline Size of aneurysm & $3(7.50 \%)$ \\
\hline Bottleneck & $5(12.50 \%)$ \\
\hline Width of aneurysm' neck & $11(27.50 \%)$ \\
\hline Previous neurosurgical clipping & $7(17.50 \%)$ \\
\hline Presence of arteries arising from the dome & $5(12.50 \%)$ \\
\hline Difficult morphology & $9(22.50 \%)$ \\
\hline
\end{tabular}

Table 4. Localisation of aneurysms

\begin{tabular}{|c|c|c|}
\hline $\begin{array}{c}\text { Complex intracranial } \\
\text { aneurysms }\end{array}$ & Localisation & $\begin{array}{c}\text { Non-complex } \\
\text { intracranial aneurysms }\end{array}$ \\
\hline $22(55.00 \%)$ & ICA & $139(44.27 \%)$ \\
\hline $8(20.00 \%)$ & AComA & $71(22.61 \%)$ \\
\hline $4(10.00 \%)$ & MCA & $58(18.47 \%)$ \\
\hline $6(15.00 \%)$ & BA & $31(9.78 \%)$ \\
\hline $0(0.00 \%)$ & VA & $9(2.86 \%)$ \\
\hline $0(0.00 \%)$ & PCA & $5(1.59 \%)$ \\
\hline $0(0.00 \%)$ & PComA & $1(0.32 \%)$ \\
\hline
\end{tabular}

ICA - internal carotid artery, AComA - anterior communicating artery, MCA - middle cerebral artery, BA - basilar artery, VA - vertebral artery, PCA - posterior cerebral artery, PComA posterior communicating artery

Table 5. Comparison of intraoperative complications in complex intracranial aneurysms (CIA) and non-complex intracranial aneurysms (nCIA) group

\begin{tabular}{|l|c|c|c|}
\hline Intraoperative complication & CIA & nCIA & $p$ \\
\hline Haematoma at injection site & $1(2.5 \%)$ & $10(3.18 \%)$ & $>0.05$ \\
\hline Ischemic stroke & $2(5.0 \%)$ & $10(3.18 \%)$ & $>0.05$ \\
\hline $\begin{array}{l}\text { Rupture of aneurysm during } \\
\text { procedure }\end{array}$ & $1(2.5 \%)$ & $10(3.18 \%)$ & $>0.05$ \\
\hline Intracranial haematoma & $0(0.0 \%)$ & $3(0.96 \%)$ & $>0.05$ \\
\hline
\end{tabular}

for the CIA than the nCIA population: 31 cases $(77.50 \%)$ for the first group and 271 cases $(88.30 \%)$ for the second group, but the difference was not significant $(p>0.05)$. Independently of the type of aneurysm, patients were hospitalised for comparable periods of time $(p>0.05)$. Mean duration of stay on the ward was 7.13 days $(\sigma=6.99)$ after endovascular embolisation of CIA and $8.74(\sigma=12.53)$ for patients with nCIA.

Out of the all 354 endovascular procedures that were analysed by our team, in 32 cases the procedure had to be aborted. The known reasons for terminating the endovascular treatment are listed in Table 6.

In the group of patients with CIA $25.00 \%$ of procedures were aborted, compared to $7.00 \%$ in the group 
Table 6. Causes of termination of endovascular treatment

\begin{tabular}{|l|c|c|}
\hline $\begin{array}{l}\text { Termination of endovascular } \\
\text { treatment - causes }\end{array}$ & $\begin{array}{c}\text { Number } \\
\text { of cases }\end{array}$ & $\begin{array}{c}\text { Number of cases } \\
\text { concerning CIAs }\end{array}$ \\
\hline $\begin{array}{l}\text { Rupture of the aneurysm sack } \\
\text { during the placement of coils }\end{array}$ & 4 & - \\
\hline $\begin{array}{l}\text { Coil evacuated from the aneurysm } \\
\text { sack and demanding removal }\end{array}$ & 1 & - \\
\hline $\begin{array}{l}\text { Failure of catheter placement } \\
\text { inside the lumen of aneurysm sack }\end{array}$ & 7 & 6 \\
\hline Spastic reaction of the artery & 8 & - \\
\hline $\begin{array}{l}\text { Insufficient contrasting } \\
\text { of the aneurysm }\end{array}$ & 2 & 2 \\
\hline Premature coil detachment & 1 & - \\
\hline Failure of stent placement & 2 & 1 \\
\hline Failure of coil placement & 1 & 1 \\
\hline Equipment failure & 1 & - \\
\hline Insufficient data & 5 & - \\
\hline Total & 32 & 10 \\
\hline
\end{tabular}

CIAs - complex intracranial aneurysms

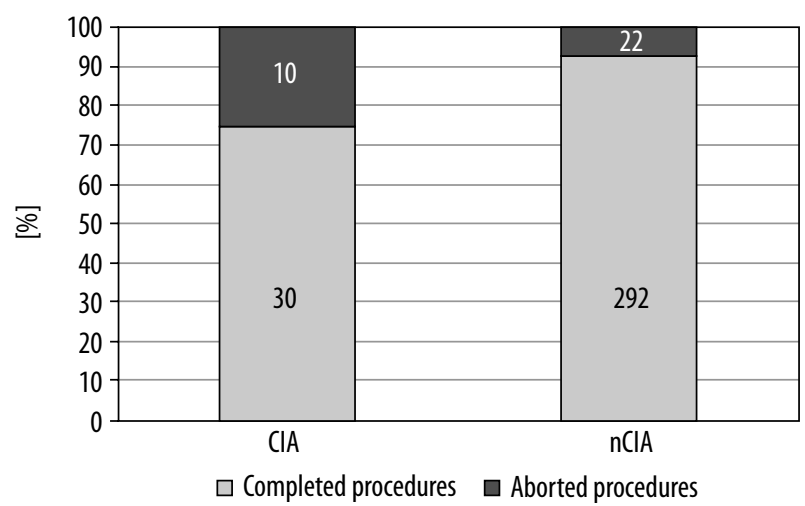

Figure 1. Comparison of prevalence of aborted procedures in complex intracranial aneurysms (CIA) and non-complex intracranial aneurysms ( $\mathrm{nCIA})$ groups

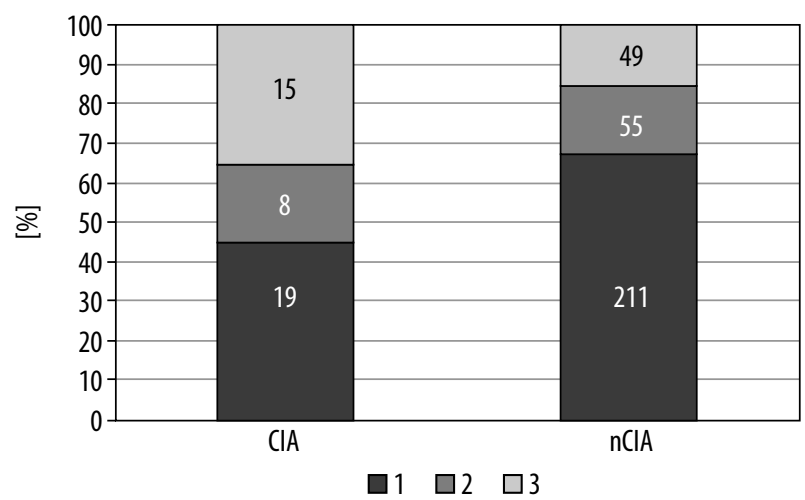

Figure 2. Montreal scale results in patients with complex intracranial aneurysms (CIA) and non-complex intracranial aneurysms ( $\mathrm{nCIA}$ )
Table 7. Results of endovascular procedures assessed by Montreal Scale

\begin{tabular}{|c|c|}
\hline Montreal Score & Number of patients \\
\hline 1 & 230 \\
\hline 2 & 63 \\
\hline 3 & 64 \\
\hline
\end{tabular}

with nCIA. The difference was statistically significant $(p=0.000191)$. It is shown in Figure 1.

The embolisation results, assessed with a use of the Montreal scale, are presented in Table 7. The mean score of all of the patients treated with endovascular approach was $1.54(\sigma=0.78)$.

Efficacy of endovascular procedures was worse in the CIA group ( $p=0.000847)$, with mean Montreal score 1.90 $(\sigma=0.91)$. In comparison, nCIA patients were assessed with mean Montreal score of $1.49(\sigma=0.75)$ (Figure 2).

In our unit, the technique of stent-assisted coiling is used relatively often. However, the presence of complex morphology of an aneurysm had no influence on the frequency of stent usage. CIA were treated with stent assistance in $57.00 \%$ of cases. nCIA patients required the placement of stent in $46.50 \%$ of interventions, and the difference was insignificant $(p>0.05)$. Only in the selected subgroup of patients suffering from subarachnoid haemorrhage (SAH) was the difference of the frequency of stenting in patients with CIA and nCIA close to statistical significance $(p=0.058125)$.

\section{Coexistence of the complex intracranial aneurysm and SAH}

SAH was observed in 84 patients, including 77 cases of nCIA and seven cases of CIA. We observed the greatest differences in subgroups of patients with CIA and nCIA in cases where aneurysms coexisted with a SAH on admission. Patients with nCIA and SAH had a mean score of $2.56(\sigma=1.04)$ in Hunt and Hess scale, while patients with CIA and SAH had a worse clinical condition and their mean Hunt and Hess score was $3.00(\sigma=1.67)$. However, the difference was insignificant $(p>0.05)$.

Patients with CIA and SAH had significantly $(p=0.041895)$ higher Montreal scale scores, with mean value of $2.14(\sigma=0.90)$, compared to $1.50(\sigma=0.72)$ for nCIA and SAH patients. It is a similar result to that obtained from analogous analysis for the entire studied population.

The clinical outcome assessed on discharge using GOS also differed in patients who suffered from SAH with CIA and nCIA. SAH-CIA patients had mean GOS of $2.86(\sigma=1.77)$, and it was significantly $(p=0.036269)$ worse than SAH-nCIA patients with mean GOS of 4.06 $(\sigma=1.39)$. It is worth emphasising that the result of comparison of clinical outcome for SAH patients only does not match the result of the same comparison for the entire studied population. 


\section{Discussion}

CIA are characterised as one of the most technically challenging intracranial vascular malformations [12-14]. CIA should be considered as more unfavourable according to difficulties in exclusion from circulation and subsequently higher risk of rupturing, resulting in $\mathrm{SAH}$ [15]. Although many published dissertations refer to CIA, there are still no established unified criteria by which aneurysm could be classified as an aneurysm of complex morphology. As Andaluz and Zuccarello observed in an article published in 2011, we can observe a literature deficiency regarding this subject even at present [11]. Attempts to classify aneurysm as CIA are rather subjective in particular studies.

Features commonly recognised in association with CIA are: giant aneurysms, localisation of difficult or morbid access, broad neck, branches arising from aneurysms, parental artery incorporation, blister-like or dissecting aneurysms, calcification of the aneurysmal wall, intraluminal thrombus, absence of collateral circulation, embedding on surrounding brain, brainstem, and cranial nerves, and previous treatments $[8-12,16,17]$. Including only patients undergoing endovascular treatment in this study determined that not all of the above-mentioned criteria could be applied. Moreover, in our clinic 3-D DSA is standard imaging method, so not all of the patients undergo CT angiography. Therefore, criteria requiring CT angiography, e.g. calcification of the aneurysmal wall, could not be used.

Applying our criteria, we extracted the group of patients with CIA accounting for $11.30 \%$. It corresponds to values presented by Andaluz and Zuccarello (9.68\%) [11]. This study showed that more frequently aborted procedures concerned patients with CIA, which is related to worse Montreal scores in this group. Therefore, patients with CIA more often cannot be secured against rupture of aneurysm and eventual complications such as long-term cognitive impairments with an effect on functional status, quality of life, and in the worst case - death [15]. Based on these data, we consider that our criteria are appropriate for describing CIA because they allow identification of aneurysms with possible technical difficulties in endovascular procedures.

Our study shows high percentages of aborted procedures in both CIA and nCIA groups. It is caused by broad inclusion criteria; because the technical difficulties of the procedure were carefully investigated, every attempt to perform the procedure was taken into consideration. In our study, even insufficient contrasting of the aneurysm or parenting vessels, failure of catheter placement, or equipment failure were counted as aborted procedures. In other studies, similar circumstances exclude it from further investigation. Therefore, comparing our high percentage of aborted procedures with other studies cannot be adequate.
In this study patients with CIA were characterised with the same values of complication ratio, GOS scores, and mean after-treatment hospitalisation time as nCIA patients. According to these data, we can reason that patients in both groups can be treated with the same safety profile but, as mentioned above, with different efficacy. Because CIA consists of aneurysms linked to worse clinical outcome, such as giant aneurysms, these results are better than could be expected [18].

It is noticeable that not many studies concerning CIA focus on SAH patients [11]. Becuase this group is considered to be more at risk of developing complications and increased mortality, the presence of additional risk factors, such as CIA, could be particularly unfavourable $[15,19,20]$. Therefore, corresponding analysis for SAH patients only was performed in our study. In parallel with the main analysis, procedures on patients with SAH and CIA were more frequently aborted and resulted in worse Montreal scores than in nCIA-SAH patients. In contrast to the main analysis, CIA-SAH patients had worse clinical outcome in GOS than nCIA-SAH patients. Therefore, co-occurrence of CIA and SAH results in decreased safety of the procedure. In qualification to invasive procedures in patients with SAH, complexity criteria should be emphasised to ensure that patients receive adequate and safe treatment.

\section{Conclusions}

To conclude, we think that the criteria of CIA proposed in this study: giant aneurysms, unfavourable bottlenecks, small width of aneurysm neck, previous neurosurgical clipping, presence of arteries arising from the dome, or difficult morphology should be taken into consideration during diagnosis and qualification to invasive treatment of intracranial aneurysms. It would help to assess features of the aneurysm, showing the possible difficulties during treatment, which were earlier unrecognised. Classifying aneurysm as CIA is related to greater possibility of aborting endovascular procedure due to technical difficulties. Simultaneously, any other criteria proposed by other authors, excluded in this study, could also be useful. Nevertheless, further studies on this subject should be performed to unify the criteria of CIA.

Additionally, if the aneurysm could be classified as CIA based on our criteria, the efficacy and safety of endovascular procedures should be broadly investigated. In doubtful cases, we suggest consideration of other invasive treatment methods, e.g. microsurgical clipping, bypass surgery, and other methods of proven efficacy, which might be beneficial for a patient [21-23].

\section{Conflict of interest}

The authors declare that there is no conflict of interest. 


\section{References}

1. Wiebers DO, Whisnant JP, Huston J 3rd, et al. International Study of Unruptured Intracranial Aneurysms Investigators. Unruptured intracranial aneurysms: natural history, clinical outcome, and risks of surgical and endovascular treatment. Lancet 2003; 362: 103-110.

2. Pierot L, Wakhloo AK. Endovascular treatment of intracranial aneurysms: current status. Stroke 2013; 44: 2046-2054.

3. Kivipelto L, Niemelä M, Meling T, et al. Bypass surgery for complex middle cerebral artery aneurysms: impact of the exact location in the MCA tree. J Neurosurg 2014; 120: 398-408.

4. Yang K, Ahn JS, Park JC, et al. The efficacy of bypass surgery using a short interposition graft for the treatment of intracranial complex aneurysm. World Neurosurg 2015; 83: 197-202.

5. Abla AA, Lawton MT. Anterior cerebral artery bypass for complex aneurysms: an experience with intracranial-intracranial reconstruction and review of bypass options. J Neurosurg 2014; 120: 1364-1377.

6. Qureshi AI, Abou-Chebl A, Jovin TG. Qualification Requirements for Performing Neurointerventional Procedures: A Report of the Practice Guidelines Committee of the American Society of Neuroimaging and the Society of Vascular and Interventional Neurology. J Neuroimaging 2008; 18: 433-447.

7. Seibert B, Tummala RP, Chow R, et al. Intracranial Aneurysms: Review of Current Treatment Options and Outcomes. Front Neurol 2011; 2: 45.

8. Fernandes ST, Alves RV, Dória-Netto HL, et al. Treatment of complex intracranial aneurysm: Case report of the simultaneous use of endovascular and microsurgical techniques. Surg Neurol Int 2016; 7: 1060-1064.

9. Hanel RA, Spetzler RF. Surgical treatment of complex intracranial aneurysms. Neurosurgery 2008; 62: 289-99.

10. Barrow DL, Cawley C. Surgical management of complex intracranial aneurysms. Neurol India 2004; 52: 156-162.

11. Andaluz N, Zuccarello M. Treatment Strategies for Complex Intracranial Aneurysms: Review of a 12-Year Experience at the University of Cincinnati. Skull Base 2011; 21: 233-242.

12. Shi X, Qian H, Fang T, et al. Management of complex intracranial aneurysms with bypass surgery: a technique application and experience in 93 patients. Neurosurg Rev 2015; 38: 109-119.
13. Abdulrauf SI, Urquiaga JF, Patel R, et al. Awake High-Flow Extracranial to Intracranial (HFEC-IC) Bypass for Complex Cerebral Aneurysms: Institutional Clinical Trial Results. World Neurosurg 2017; https://doi.org/10.1016/j.wneu.2017.04.016.

14. Labeyrie PE, Gory B, Aguilar-Perez M, et al. The pCONus Device for Treatment of Complex Wide-Neck Anterior Communicating Artery Aneurysms. World Neurosurg 2017; http://dx.doi. org/10.1016/j.wneu.2017.02.045.

15. Suarez JI, Tarr RW, Selman WR. Aneurysmal Subarachnoid Hemorrhage. N Engl J Med 2006; 354: 387-396.

16. Jeon HJ, Kim BM, Kim DJ, et al. Combination of Multicatheter Plus Stent or Balloon for Treatment of Complex Aneurysms. AJNR Am J Neuroradiol 2016; 37: 311-316.

17. Esposito G, Regli L. Surgical decision-making for managing complex intracranial aneurysms. Acta Neurochir Suppl 2014; 119: 3-11.

18. Ha SW, Jang SJ. Clinical Analysis of Giant Intracranial Aneurysms with Endovascular Embolization. J Cerebrovasc Endovasc Neurosurg 2012; 14: 22-28.

19. Tykocki T, Czyż M, Machaj M, et al. Comparison of the timing of intervention and treatment modality of poor-grade aneurysmal subarachnoid hemorrhage. Br J Neurosurg 2017; doi: https://doi.org/10. 1080/02688697.2017.1319906.

20. Petridis AK, Kamp MA, Cornelius JF, et al. Aneurysmal Subarachnoid Hemorrhage. Dtsch Arztebl Int 2017; 114: 226-236.

21. Breu AK, Hauser TK, Ebner FH, et al. Morphologic and Clinical Outcome of Intracranial Aneurysms after Treatment Using Flow Diverter Devices: Mid-Term Follow-Up. Radiol Res Pract 2016; 2016: 2187275.

22. Jin SC, Kwon DH, Song Y, et al. Multimodal Treatment for Complex Intracranial Aneurysms: Clinical Research. J Korean Neurosurg Soc 2008; 44: 314-319.

23. Ismail Alhothi A, Qi T, Guo S, et al. Neuroform stent-assisted coil embolization: a new treatment strategy for complex intracranial aneurysms. Results of medium length follow-up. Neurol Neurochir Pol 2010; 44: 366-374. 